\title{
Addressing Accurate Trace Element Analysis at High Spatial Resolution in EPMA
}

\author{
M.J. Jercinovic*, M.L. Williams*, J. Allaz*, J.J. Donovan** \\ *Department of Geosciences, University of Massachusetts, Amherst, MA 01003 **CAMCOR, University \\ of Oregon, Eugene, OR 97403
}

The application of the electron microprobe to trace element analysis of sub-micron sized domains is becoming more prevalent as recent technological developments allow improved beam and spectrometer performance at lower voltage through a broad range of current than would previously have been deemed realistic [1]. High spatial resolution EPMA mapping reveals important details of the compositional domain structure of materials, commonly revealing micro-or nano-scale heterogeneity and necessitating a more detailed analytical approach. In particular, high $\mathrm{Z}$ phases limit electron scattering (in bulk analysis) to the extent that the analytical spatial resolution can be improved by achieving beam diameters down to $80100 \mathrm{~nm}$, now attainable with use of high brightness sources. Scattering effects generally prohibit further improvement (i.e. too much below 100nm) for practical analysis in bulk EPMA, with the exception of very special cases. Spectrometers with high X-ray collection efficiency, and software strategies such as count integration from multiple spectrometers permit the use of lower beam current and voltage (where appropriate) to further decrease the scattering volume dimensions, beam diameter and ultimate analytical spatial resolution while still allowing sufficient count precision for trace element analysis. However, along with these improvements in beam quality and counting efficiency come potential difficulties. These have not been recognized or are considered negligible in major element analysis, but become substantial in trace element micro-analysis. Accuracy in this realm is critically a function of: 1) The characterization and precise measurement of background; 2) effects of fluorescence at a distance; and 3) possible beam damage along with current and count stability accompanying the necessary lengthy count times and high current density.

Background -Background measurement must be exceptionally precise and accurate for trace element microanalysis. As peak/background approaches unity, background measurement methodology requires consideration and validation comparable to that for peak acquisition. Even the most precise acquisition will be inaccurate if interferences and background shape effects are not correctly identified and addressed [2]. Detailed WDS scanning is essential for basic characterization of the background, but if the required sensitivity is on the order of a few tens of ppm, attaining adequate precision in the scan may be impractical or even introduce additional error (through beam damage, etc.). A new approach, the multi-point method, now available via Probe for EPMA [3], relies on precise measurement of up to 24 points throughout an appropriate region of the spectrum, then regression to establish the curvature while identifying interferences, eliminating those points with values statistically outside the best-fit (Fig. 1). In this way, the correct background intensity beneath the peak can be accurately attained, and obvious interferences in the background spectrum can be accounted for.

Fluorescence -Accuracy in trace element analysis is strongly affected by the consequences of fluorescence. In some instances, effects occurring outside of the direct excitation volume can result in inaccuracy [4]. A sub-micron analytical spatial resolution can theoretically be achieved in monazite for trace-level $\mathrm{PbM} \alpha$ analysis [2], but this does not consider the possible effects of compositional variation adjacent to the volume of direct electron-induced X-ray emission. In particular, phases containing significant proportions of heavy elements (e.g., monazite) have the potential to produce relatively high energy, and high intensity ionizing radiation (both characteristic and continuum) at considerable distance from the beam (Fig. 2). This effect may result in spurious apparent concentrations if the element of interest is concentrated in the adjacent phase, for example, tens of ppm of apparent Th in a Th blank five microns from a boundary with $6 \mathrm{wt} . \% \mathrm{Th}$, or may also result in unexpected interferences on peak and 
background positions $[2,5]$. The multipoint approach to background acquisition (above) can account for these background interferences. Fluorescence effects should also be considered when comparing standard intensities, obtained from compositionally homogeneous materials (a portion of the std. intensity can arise from fluorescence several microns or more distant) to unknowns exhibiting micro-scale heterogeneity. If recognized and properly characterized, such effects can, in some cases, be adequately accounted for in order to give a more accurate final result [6].

Current Density -High spatial resolution trace element analysis implies exceptionally high beam current density during lengthy count times [5]. Phases traditionally considered to be robust during EPMA may be less stable at high current density for the lengthy count times required for the precision objective. More beam-sensitive materials (e.g. phosphates, carbonates, glasses), challenging even under modest conditions, may be strongly affected. For example, phosphorous loss during beam exposure can result in monazite during high-current EPMA [5]. Absorbed current behavior suggests that internal space charge effects can be significant in monazite and carbonate (Fig. 3). Coating materials and thickness, time-dependent intensity acquisition, and alternating on-off peak acquisition methods offer ways to mitigate these effects.

References

[1] M.J. Jercinovic, et al., Microsc. Microanal. 14, (Suppl. S2) (2008) 1272-1273.

[2] M.J. Jercinovic, et al., Chem. Geol. 254 (2008) 197-215.

[3] Probe Software, Inc., Eugene, OR, USA

[4] S.J.B. Reed, Electron Microprobe Analysis, Cambridge University Press, 1993.

[5] M.J. Jercinovic and M.L. Williams, American Mineralogist 90 (2005) 526-546.

[6] J.A. Escuder et al., 11 th EMAS Workshop. IOP Conf. Series: Mat. Sci. Eng. 7 (2010). doi:10.1088/1757-

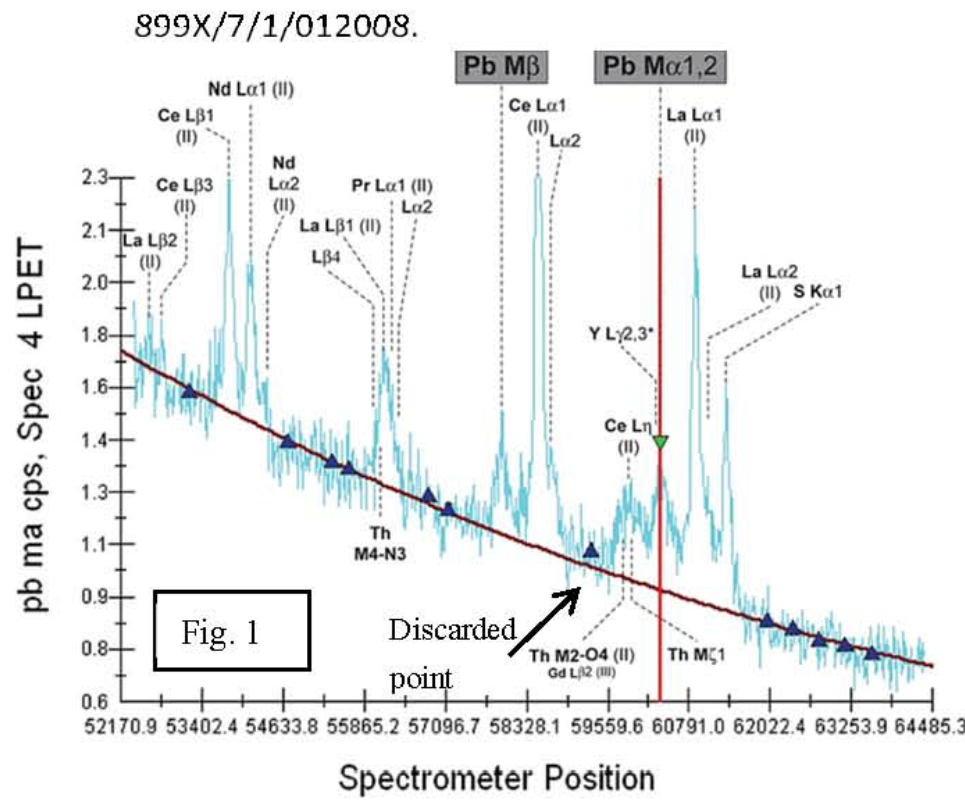

Fig. 1 (above). Spectrum (PET) of Pb-Ma region in monazite. Multipoint background acquisition (triangles) can be regressed and evaluated to yield proper curvature (dark line). Fig. 2 (upper-rt.). X-ray range for LREE and fluorescence intensity vs. distance in monazite. Inset schematic illustration of analysis of trace element in phase $\mathrm{A}$ that is concentrated in phase B. Fig. 3 (rt.) Ca Ka intensity with time in $\mathrm{CaCO} 3$, 20um, 200nA, 15kV. Compare to absorbed current measurement.
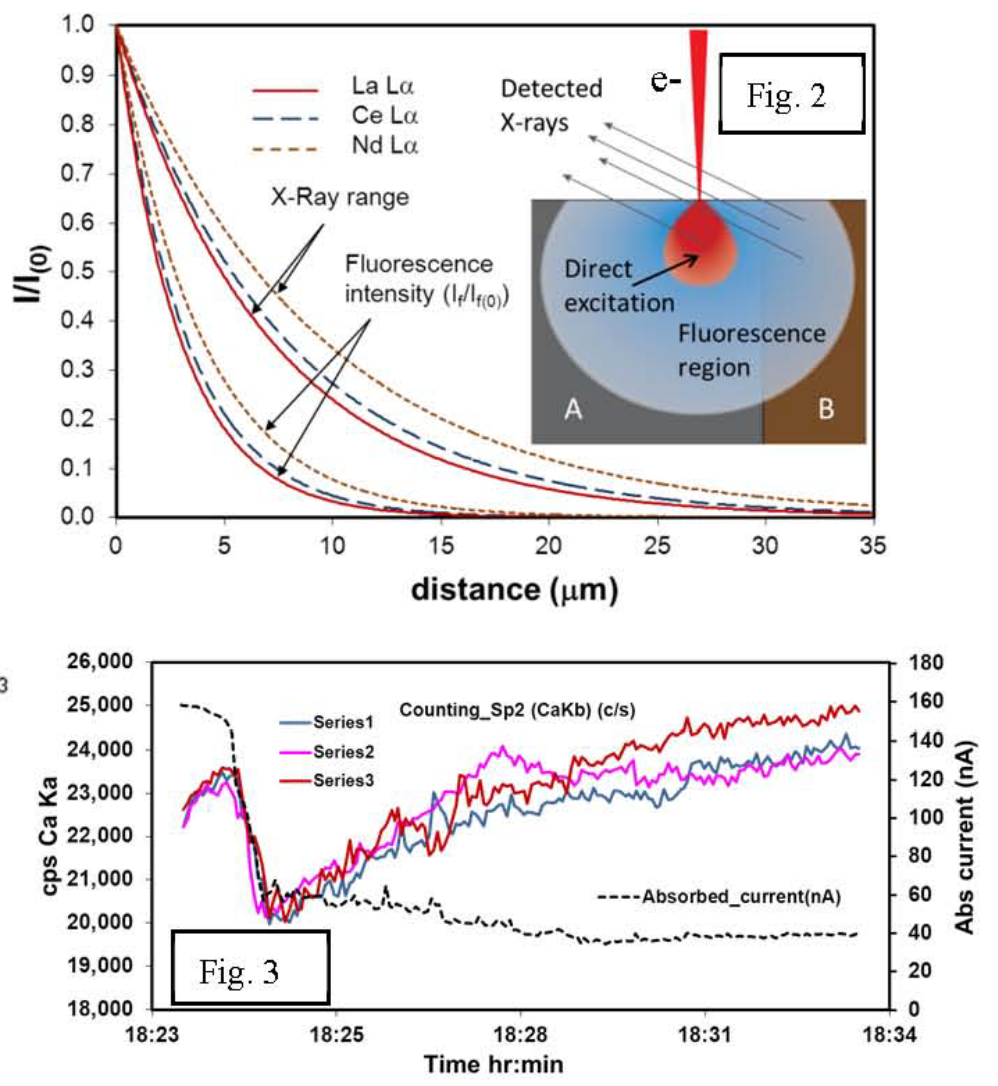